\title{
A New Method to Detect Gastro-esophageal Reflux in Critically III Mechanically Ventilated Patients: A Clinical Proof of Concept Study
}

YARON Phillip Bar-Lavie ( $\nabla$ y_barlavie@rambam.health.gov.il)

Critical Care Medicine, Rambam Medical Center, Haifa, Israel https://orcid.org/0000-0001-6846-0538

Shirly Steinlauf

ART MEDICAL

\section{Research}

Keywords: Gastro-esophageal Reflux, Aspiration Pneumonia, Ventilator Associated Pneumonia, Gastric Feeding Tube, Feeding tube insertion

Posted Date: December 18th, 2020

DOI: https://doi.org/10.21203/rs.3.rs-127678/v1

License: @ (i) This work is licensed under a Creative Commons Attribution 4.0 International License. Read Full License 


\section{Abstract}

Background

Tracheal aspiration of gastric content among mechanically ventilated and tube-fed critically ill patients is of great concern. The mechanism leading to bacterial colonization of the lower respiratory tract in mechanically ventilated patients is tracheal aspiration of oral or gastric contents leading to Ventilator Associated Pneumonia (VAP). VAP is the most common nosocomial infection in the Intensive Care Unit (ICU). ICU-VAP generates about half of the cases of hospital-acquired pneumonia. There are various nursing practices available to reduce the rate of aspiration, but their efficacy is limited and they are not strong enough to prevent aspiration and VAP in practice. In this clinical study we used the smARTrack System, by ART MEDICAL, to assess the frequency of gastro-esophageal reflux in 20 ventilated ICU patients and its' correlation to procedures during routine patient care.

\section{Methods}

The smARTrack System is comprised of a novel gastric tube and an electronic monitoring console, which contains the system's software. The tube contains a series of bio-impedance sensors that can detect the presence of fluid around them. We conducted a study on 20 ICU ventilated patients at the Rambam Health Care Campus in Haifa, Israel. Reflux events and their duration were recorded when the tube detected gastric content rising above the sensors located at the lower esophageal sphincter

Results

Our main findings shows that gastro-esophageal reflux occurs on average three times per hour of mechanical ventilation. We have also recorded three common clinical scenarios that were associated with a high risk of reflux; patient position-change, enteral fluid bolus, tracheal suction and migration of the feeding tube tip during ongoing use.

\section{Conclusions}

Gasto-esophageal reflux is a common event in the ICU as shown by the smARTrack System and may be related to routine patient care procedures. This study provides an important insight into the consequences of these procedures and might suggest ways to further improve them in reducing the rate of gasto-esophageal reflux and the risk of VAP among mechanicaly ventilated ICU patients.

https://clinicaltrials.gov/ct2/show/NCT02705781?term=smARTrack\&draw=2\&rank=1 registered 11.3.2016

\section{Introduction}

Tracheal aspiration of gastric content among mechanically ventilated and tube-fed critically ill patients is of great concern [1].

The mechanism leading to bacterial colonization of the lower respiratory tract in mechanically ventilated patients is tracheal aspiration of oral and-or gastric contents leading to Ventilator Associated Pneumonia (VAP). VAP is the most common nosocomial infection in the Intensive Care Unit (ICU) [2] ICU-VAP generates about half of the cases of hospital-acquired pneumonia [2-4].

The CDC suggested that valid and accurate surveillance data is mandatory to assess the effectiveness of VAP prevention strategies. They noted that effective measures to improve mechanically ventilated patient outcomes did not target pneumonia prevention [5]. There are various nursing practices that may help reduce the rate of aspiration and VAP, but their efficacy is limited and their implementation is limited. These practices include elevation of the head of the bed to 30-45 degrees, mouth washing with chlorhexidine and frequent suctioning, and oral or gastric decontamination.[6] Some recent technologies have been proposed to decrease gastric content reflux to aspirate into the lungs with relative efficacy. Tracheal tubes with sub-glottic aspiration (a), CO2 guided sealing of the cuff (b), new nasogastric tubes (e motion (c), Lunguard (d) are all technologies that have shown some decrease in tracheal aspiration.

In addition in tube-fed patients, the process of tube insertion and accurate placement is of importance. Insertion of a Gastric Feeding Tube (GFT) can beperformed blindly at the bedside, by $\mathrm{CO} 2$ guided method [7], by Comtrack technique [8] or with magnetic , fluoroscopic or endoscopic guidance into the stomach $[9,10]$. Although generally safe and effective, there is a wide spectrum of known complications. The most common serious complication is misplacement of the feeding tube into the bronchial tree with resulting pneumonitis, pneumonia, and/or pneumothorax if not recognized. The incidence of this event is $2.4 \%-3.2 \%$ of nasogastric tube insertions [11]. Positioning complications may also occur throughout the duration of use of the tube. Movement of the distal tip may occur even when the proximal GFT is taped in place. This is most likely to occur with soft, small-bore GFTs. Currently, Xray imaging is the gold standard for documenting the position of GFTs [12]. However, it is not routinelyused in some countries due to the risk of radiation exposure, cost and delay of feeding [13-14]. Moreover, the National Patient Safety Agency drew attention to the risk of misinterpretation of Xrays used to confirm GFTs position. [15]

To our knowledge, there are currently no devices providing a GFT that monitors reflux episodes and/or detects tube migration during ongoing use. Thus, a GFT that enables a safer placement procedure, detects reflux as it occurs and may prevent pneumonia related to gastric content aspiration can be of help for a better medical care. 
We present our first experience in ICU patients, with the smARTrack ${ }^{\mathrm{TM}}$ system, a novel method to detect gastro-esophageal reflux in critically ill, mechanically ventilated patients, as a proof of concept study. The aim of our study was to assess the functionality and accuracy of the smARTrack ${ }^{\text {TM }}$ system to guide operators in the placement procedure, to detect gastro-esophageal reflux and its' correlation to procedures of routine patient care.

\section{Materials And Methods}

The smARTrack ${ }^{\mathrm{TM}}$ System is comprised of a novel gastric tube and an electronic monitoring console which contains the system's software. The GFT contains a series of bio-impedance sensors that can detect the presence of fluid around them. The console serves as the guiding interface to the user during initial tube placement, and monitors in real time, the tubes' position (Fig. 1). Once the system senses that the tube has moved from its correct position or when massive reflux is detected, an alarm is activated, and feeding is automatically stopped.

\section{Study Design}

We conducted a study on 20 ICU ventilated patients at the Rambam Health Care Campus in Haifa, Israel. The study was approved by the local hospital Ethics Committee (Helsinki, registration number: 0392-11-TLV and ClinicalTrials.gov: NCT02705781). Informend concent was given by $1^{\text {st }}$ degree next of kin and approved by independent physician. Inclusion criterias were: to be at least 18 years old, expected to be mechanically ventilated and to receive enteral feeding for the 72 hours of the study period. Exclusion criteria were recent abdominal surgery and any known abnormalities or diseases of the esophagus or stomach.

\section{Study Procedures}

The GFT was placed via the naso-gastric route. Insertion of the tube to gastric position were guided by the smARTrack console screen. Correct tube position was confirmed by upper abdominal X-ray. Enteral nutrition was delivered by a feeding pump (Alcore Sentinel, Smithfield, USA) according to the regimen prescribed by the department dietitian and the medical team. Reflux events and their duration were recorded when the tube detected gastric content rising above the sensors located at the lower esophageal sphincter. In each reflux event, the console mechanics clamps the feeding bag tubing causing the pump to sense occlusion and stop feeding. Feeding by the pump is resumed when the tube sensors were cleared, detecting that reflux has resolved. If the system was alerting that tube displacement occurred, the need for tube repositioning is evaluated and performed. Patient care and feeding processes were video-recorded throughout the study period. Video-recorded clinical procedures (such as position change, enteral fluid bolus, tracheal suction) were later correlated to reflux and out-of-position events, as documented by the system console.

\section{Results}

Twenty patients were included in the study. There were no dropouts. The average time for tube insertion was 4 minutes (range 1-10). In three patients, the system alerted for malposition during the initial placement procedure: twice due to suspected tracheal insertion and once due to curling in the patients' mouth.

The smARTrack ${ }^{\mathrm{TM}}$ Feeding System was connected to the 20 patients for a cumulative total period of 57 days (average 2.86 days per patient (range $2-3$ days)).

Frequency and duration of reflux events:

4072 reflux events were documented (average 204 per patient, range 40-570, median-183, s.d.-117). This leads to a patient average of 3 reflux events per hour of mechanical ventilation (range 0-42, median-3, s.d.- 3.8). (Table 1)

Table 1: patient reflux frequency

\begin{tabular}{|c|c|c|c|c|c|c|c|c|c|c|c|c|c|c|c|c|c|c|c|c|}
\hline $\begin{array}{l}\text { Patient } \\
\text { No. }\end{array}$ & 1 & 2 & 3 & 4 & 5 & 6 & 7 & 8 & 9 & 10 & 11 & 12 & 13 & 14 & 15 & 16 & 17 & 18 & 19 & 20 \\
\hline $\begin{array}{l}\text { Total } \\
\text { reflux }\end{array}$ & 100 & 165 & 290 & 181 & 211 & 309 & 78 & 65 & 133 & 40 & 185 & 170 & 200 & 227 & 229 & 145 & 570 & 386 & 164 & 224 \\
\hline
\end{tabular}

About a third of reflux events lasted 3-10 seconds, more than half, lasted 10-30 seconds and 1\% lasted more than 30 seconds. Maximal reflux duration was 265 seconds (range 3-265, average 16.1sec, median-14, s.d-11.9) (fig. 2).

Most reflux events lasted up-to 30 second. Longer reflux durations were relatively rare.

\section{Gastro-esophageal reflux and its' correlation to procedures of routine patient care}

We assume that each group of procedures in the routine of patient care were executed with similar characteristics (i.e., duration, and method). 
Over a period of three enrollment days, there were 827 events of Position Change (PC). These included any significant movement in the bed during washing, bedding or seating, and during any medical or nursing related procedures. The average number of PCs was 41 per patient (range $25-85$, median-39, s.d.-13.9).

The correlation between PC and occurrence of reflux events was examined. As seen in Figure 3, most of the events occurred within 5 minutes after PC. In 827 PCs, reflux occurred within 0-5 minutes 300 times (40\%)

\section{Enteral Fluid Bolus:}

Critically ill patients, frequently receive enteral water, medication or nutrition by Fluid Boluses (FB) using syringe injections. The volume of these FBs may range between 50-60 cc and usually given by the nurse as a high pressure "Push". 151 FB events were documented in 15 patients, an average of 8 FB per patient (range 3-18, median-8, s.d-4.1). 35 reflux events that occurred within 5 minutes of FB where detected, an average of two reflux episodes per patient (range 0-7, median-1.5, s.d-1.8). Figure 5 shows patient reflux incidence after FB.

\section{Tracheal Suction:}

327 Tracheal Suction (TS) procedures were performed during the study period, an average of 17 TSs per patient (range 8-30, median-16, s.d.-7.1). In 211 TS procedures, at least one reflux event occurred in every patient within 5 minutes. As a result of TS, an average of 11 reflux events per patient was documented (range $0-28$, median -10, s.d.-8.7). Almost two thirds, (64.5\%) of TS procedures resulted in reflux events within five minutes. Figure 6 shows the percentage rate of reflux events per patient within 5 minutes of TS.

\section{Discussion}

The smARTrack ${ }^{\mathrm{TM}}$ Feeding System is a novel gastric tube and monitoring device. It was developed to overcome the complications associated with enteral nutrition in mechanically ventilated patients. After closely monitoring 20 mechanically ventilated patients for a cumulative period of 57 days, this is the first document description of the distribution of gastro-esophageal reflux in critically ill mechanically ventilated patients.

One of our main findings shows that GER occurs three times per hour of mechanical ventilation. Reflux above the LES occurred 4072 times or an average of 204 events per patient (range 40-570). We have also recorded three common clinical scenarios that were associated with a high risk of reflux. These include patient position-change, enteral fluid bolus, tracheal suction and migration of the feeding tube tip during ongoing use.

Patient position change:

The mechanically ventilated patient moves spontaneously in bed and is very often passively moved by the nursing and medical teams. Lateral rotation is done during washing, wound treatment, bedding and to prevent pressure sores. Vertical downward movement of the head of the bed occurs for various procedures such as central line placement, washing or bedding.

These events may create a gravitation gradient that promotes GER, thus raising the probability of regurgitation of gastric content and its' aspiration. In 827 (average 41) events of position change, there were 300 (40\%) reflux events, within 5 minutes. In order to prevent this sequence the patients should be treated with their torso raised to 30 degrees at least. Another reflux preventing mechanism might be to open the gastric tube to drain whenever the head of the bed is lowered to the supine position.

Enteral fluid bolus:

Fluid boluses of 50-60 ml are given by syringe to the GFT for delivery of various medications and nutrition. This procedure is usually done by the nurse as a rapid push, unaware of the steep rise in gastric pressure. Because of the positive pressure gradient, gastric content is refluxed to the esophagus. In 151 events (average 8) of enteral Fluid Boluses, 35 reflux events occurred within 5 minutes (23\%). A method to prevent this surge of gastric pressure may be to reduce bolus volume or avoid syringe boluses altogether and to deliver the fluid or medication by slow enteral infusion.

Feeding tube displacement during enteral nutrition administration:

In 5 out of the 20 patients (25\%), 7 tube tip displacement events were detected. In all of them, the tip moved proximally to the upper esophagus. Reflux and aspiration may have been prevented by occlusion of the pumps' feeding-set by the system alarm sequence.

Enteral nutrition by a feeding tube is the standard of care for most mechanically ventilated patients in the ICU and elsewhere. The passage of the tube through the esophagus to the stomach opens the LES and promotes gastro-esophageal reflux. Once the LES is passed, there is nothing but gravitation to prevent the fluid from reaching up-to the patients' mouth. When refluxed gastric content reaches the patients' mouth, there is a high risk for tracheal aspiration and as a result, a chance to develop an aspiration pneumonia called VAP. Aspiration of gastric content can occur without obvious evidence of vomiting, particularly in sedated patients or those with poor mental status, and absent gag reflex. The regurgitation is usually silent until signs of 
respiratory compromise or pneumonia develop. As mentioned before, clinically significant aspiration occurs in up to $88 \%$ of ventilated patients with tracheostomies or trans-laryngeal intubation [16]. Aspiration pneumonia adds an average of 7 days of mechanical ventilation, is associated with a very high mortality and an added average healthcare cost of $\$ 40,000$ [17]. Currently, the best-proven means of reducing the risk of VAP is by elevating the head of the bed, presumably reducing the risk of reflux, regurgitation and aspiration.

Tracheal Suction:

Tracheal Suction (TS) is a routine and common procedure in the ICU. A negative pressure of 100-700 mm mercury is applied to the airway for a period of 10-20 seconds, sometimes 2-3 times in a row, causing a fast outward air flow and a quick and significant reduction in thoracic pressure. In many units where a closed suction system is not in use, this procedure is preceded by disconnection from the ventilator, thus reducing airway pressure to zero even before onset of the suction procedure. In the ICU where the study was conducted, a closed suction system is the standard of care. Therefore, mechanical ventilation was continued during the TS procedure, thus delivering air-leak compensation by the ventilator and reducing the pressure drop. Still, TS was very often followed by reflux probably caused by a rapid pressure drop in the chest and a positive pressure gradient from the stomach to the esophagus. In 327 procedures (average 17) of TS performed, reflux occurred within 5 minutes in 211 events (64.5\%). The average was 11 reflux events per patient.

To prevent the pressure gradient during TS, it may be suggested to maintain a raised head of the bed, reduce negative suction pressure, and shorten the procedure, to perform it intermittently and to use a closed suction system so that mechanical ventilation is not interrupted.

Tube tip dislocation:

As a result of various procedures, such as patient movement or positioning change, the gastric tube may get dislodged from its correct position and move in, or outwards. The results of these tube movements may be hazardous if the tip has shifted to the upper esophagus or throat, and an aspiration may result.

Our study has several limitations: It is a pilot observational study studying the reflux of patients without comparing our technique to a gold standard methodology. Our technique will have to be compared to other methods such as tracheal detection of pepsin, stable isotopes, or addition of colorant to the enteral nutrition. We have also to consider that many of the detected reflux events may be of non-clinical significance but those of long duration and high volume may be more relevant.

\section{Conclusions}

In this proof of concept study with the smARTrack system, GFT placement and reflux detection were successfully achieved. The first contribution of this system is its' capability to guide the GFT placement procedure, continuously monitor its' position and guide repositioning if needed. Furthermore, a correlation between common clinical procedures such as position change, tracheal suction or enteral fluid bolus and reflux events was documented. The next development step will be to develop and evaluate methods to prevent reflux and also to prevent tracheal aspiration and pneumonia - once reflux was detected by the system.

\section{Declarations}

\section{Ethical Approval and Consent to participate}

The study was approved by the local hospital Ethics Committee (Helsinki, registration number: 0392-11-TLV and ClinicalTrials.gov: NCT02705781). Informed consent was given by $1^{\text {st }}$ degree next of kin and approved by independent physician.

\section{Consent for publication}

Both authors have reviewed the final manuscript and given consent to the publication.

\section{Availability of supporting data}

All patient study and clinical and laboratory data are maintained in hospital files according to IRB rules and saved for 15 years.

\section{Competing interests}

Dr. Yaron Bar-Lavie, MD. Certifies that he has NO affiliations with or involvement in any organization or entity with any financial interest, or non-financial interest in the subject matter or materials discussed in this manuscript. Director, Division of Critical Care Medicine, Rambam Health Care Campus, Israel. y_barlavie@rmc.gov.il

\section{Disclosure of Potential Conflicts of Interest}

Shirly Steinlauf, M.Sc., works for ART MEDICAL in biomedical engineering at ART MEDICAL Company. As part of her position, she conducts research in the medical and marketing fields. As part of the current manuscript, Shirly was involved in the fields of development and outline of trial protocols, trained site staff, and managed trial materials. Shirly received a salary for this work. Head of RA/QA/CA, ART MEDICAL, Netanya, Israel.

shirly.steinlauf@gmail.com. 


\section{Funding}

ART MEDICAL has provided the gastric tubes used in the study as well as the capital equipment used in the study. The hospital research fund received compensation for administrative expenses and partial employment of a study coordinator. Dr. Bar-Lavie - the primary investigator and first author received no compensation or any financial support for this research study.

\section{Authors' contributions}

YBL conceptualized the study, wrote the protocol, applied for IRB approval, recruited all the patients and received informed consent, performed all study procedures including gastric tube insertions, collected patient data and files.

YBL performed data collection and evaluation, supervised statistical analysis and wrote all parts of the manuscript.

Shirly Steinlauf assisted in protocol development, technical considerations, data organization and analysis, as well as preparation of tables and graphs based on collected data.

\section{Acknowledgements}

The authors would like to thank Prof. Pierre Singer, Director, Department of General Intensive Care and the Institute for Nutrition Research, Rabin Medical Center, Israel, for reviewing the manuscript and giving invaluable comments.

\section{References}

1. Norma A. Metheny, PhD, Ray E. Clouse, MD, Yie-Hwa Chang, Tracheobronchial aspiration of gastric contents in critically ill tube-fed patients: Frequency, outcomes, and risk factors. Crit Care Med. 2006 Apr; 34(4): 1007-1015.

2. Nosocomial infections in the intensive care unit: Incidence, risk factors, outcome and associated pathogens in a public tertiary teaching hospital of Eastern India. Sugata Dasgupta, Soumi Das, Neeraj S. Chawan, and Avijit Hazra. Indian J Crit Care Med. 2015 Jan; 19(1): 14-20.

3. American Thoracic Society, Infectious Diseases Society of America. Guidelines for the management of adults with hospital-acquired, ventilatorassociated, and healthcare-associated pneumonia. Am J Respir Crit Care Med. 2005;171:388-416.I

4. Vincent JL, Bihari DJ, Suter PM, Bruining HA, White J, Nicolas-Chanoin MH, Wolff M, Spencer RC, Hemmer M. The prevalence of nosocomial infection in intensive care units in Europe. JAMA. 1995;274:639-644.

5. CDC-2018. https://www.cdc.gov/nhsn/PDFs/pscManual/6pscVAPcurrent.pdf

6. DiBardino DM,Wunderink RG. Aspiration pneumonia: A review of modern trends. Journal of Critical Care 30 (2015) 40-48

7. Araujo-Preza CE, Melhado ME, Gutierrez FJ, Maniatis T, Castellano MA. Use of capnometry to verify feeding tube placement. Crit Care Med. 2002;30(10):2255-2259.

8. Smithard D, Barrett N, Hargroves D, Elliot S.: Electromagnetic Sensor-Guided Enteral Access Systems: A Literature Review. Dysphagia (2015) 30:275-285

9. Roberts S, Echeverria P, and Gabriel SA. Devices and Techniques for Bedside Enteral Feeding Tube Placement. Nutrition in Clinical Practice 2007; 22:412-420

10. Jinoo Kim, Ji Hoon Shin. Placement of feeding tubes using fluoroscopy guidance and over-the-wire technique: A technical review. Gastrointest Interv 2017;6:135-139

11. James L. Stayner, MD ; Akash Bhatnagar, MD ; Arlo N. McGinn ; and John C. Fang, MD. Feeding Tube Placement: Errors and Complications. 2012.

12. Nyqvist KH1, Sorell A, Ewald U. Litmus tests for verification of feeding tube location in infants: an evaluation of their clinical use. J Clin Nurs 2005;14:486-95.

13. National Patient Safety Agency (2011) Patient Safety Alert 002: Reducing the Harm caused by Misplaced Nasogastric Feeding Tubes in Adults, Children and Infants. London: NPSA.

14. National Patient Safety Agency (2005) Patient Safety Alert 05: Decreasing the Harm caused by Misplaced Nasogastric Feeding Tubes. London: NPSA.

15. National Patient Safety Agency Patient Safety Alert 002: Reducing the Harm caused by Misplaced Nasogastric Feeding Tubes in Adults, Children and Infants. London: NPSA. (2011)

16. Effectiveness of an Aspiration Risk-Reduction Protocol. Norma A. Metheny, Jami Davis-Jackson, Barbara J. Stewart. Nurs Res. 2010 ; 59(1): $18-25$.

17. Warren DK, Shukla SJ, Olsen MA, et al. Outcome and attributable cost of ventilator-associated pneumonia among intensive care unit patients in a suburban medical center. Crit Care Med.2003;31(5):1312-7. 10.1097/01.CCM.0000063087.93157.06

\section{Figures}



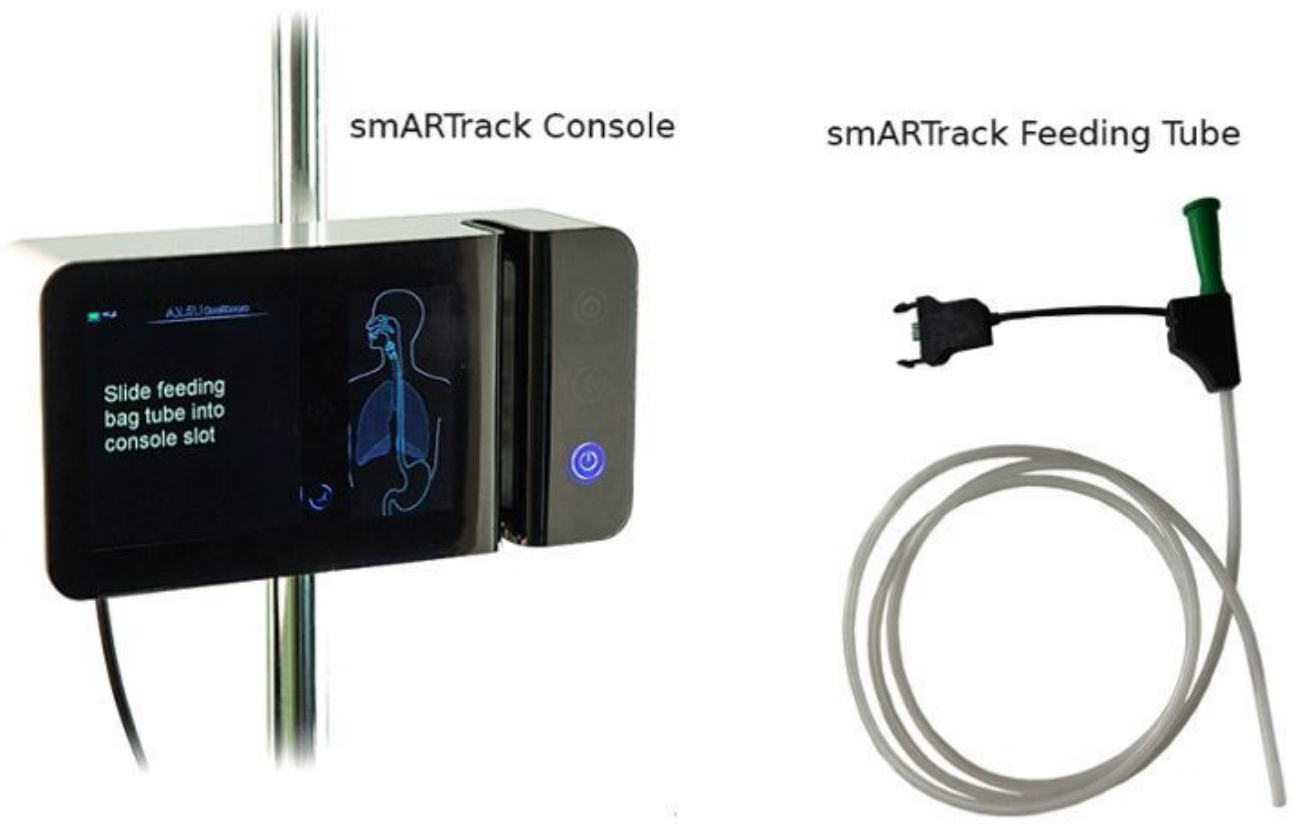

\section{Figure 1}

smARTrack System console.

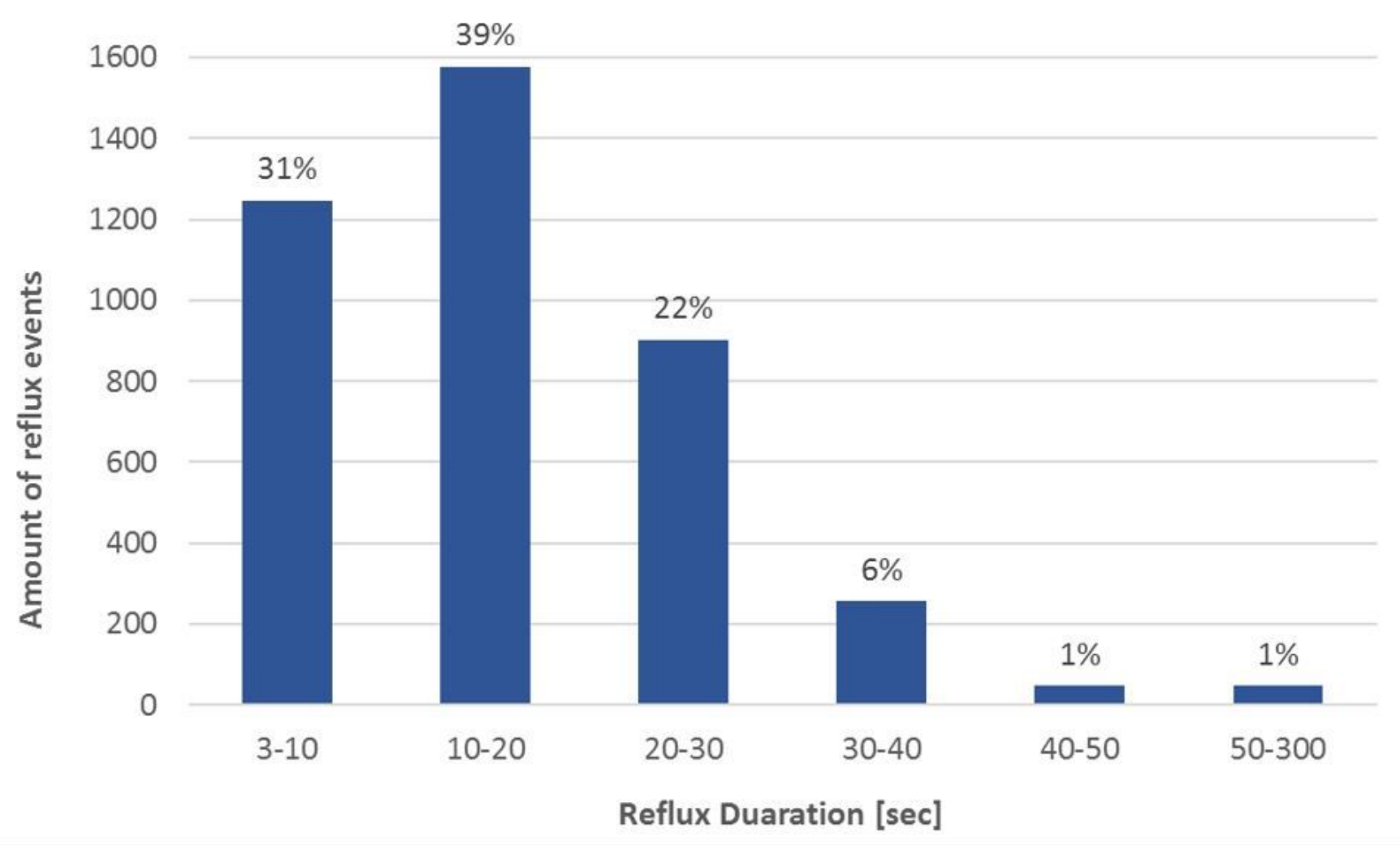

\section{Figure 2}

Distribution of reflux durations. Note: due to sensors noise, $3 \mathrm{sec}$ is the minimal reflux duration identification 


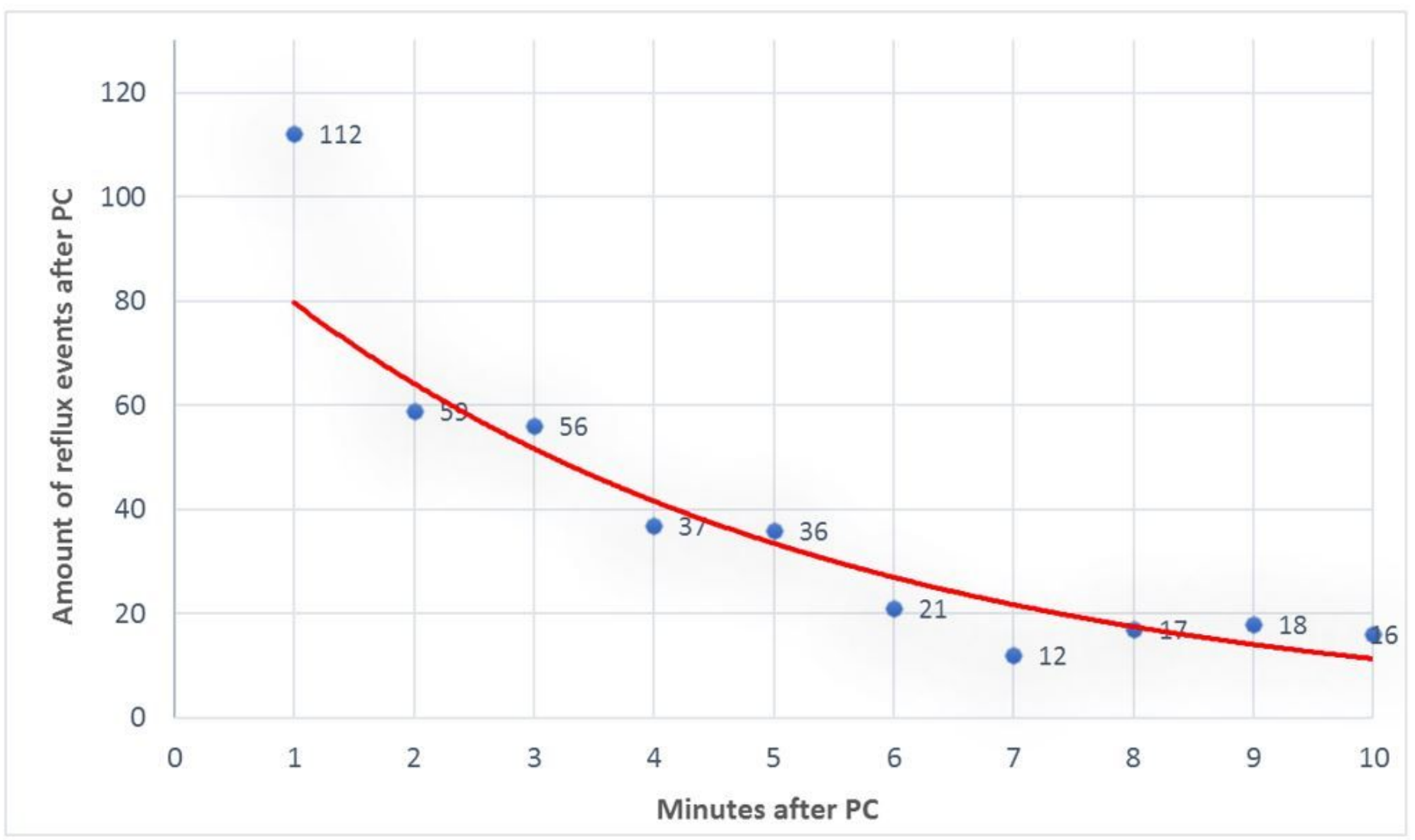

Figure 3

Time to reflux event after Position Change

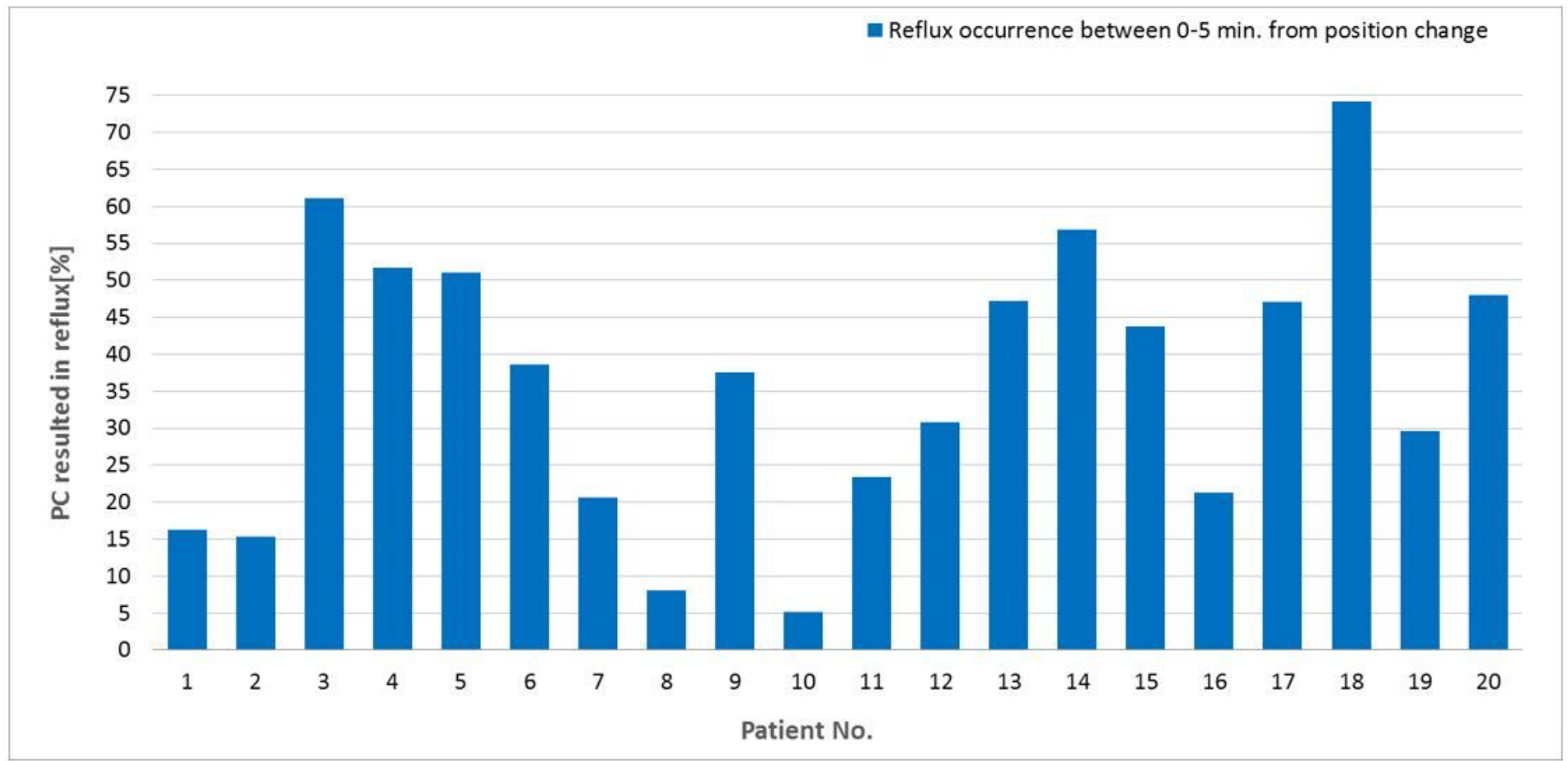

Figure 4

Time to reflux event after Position Change 


\section{Reflux within 5 min after fluid bolus}

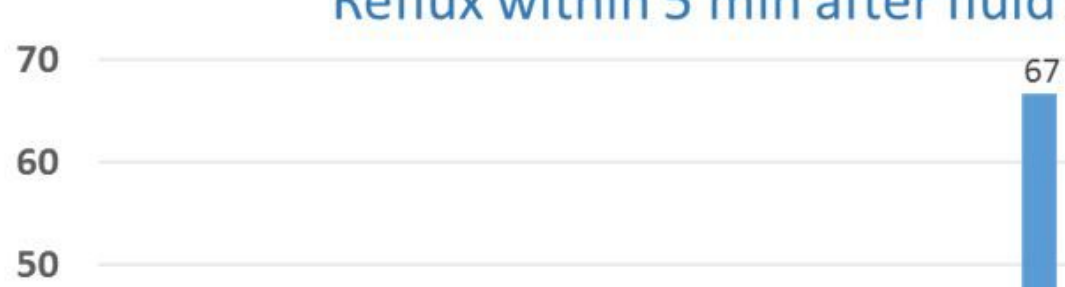

Bolus

40 resulted in reflux [\%] 30

$$
20
$$

10

0

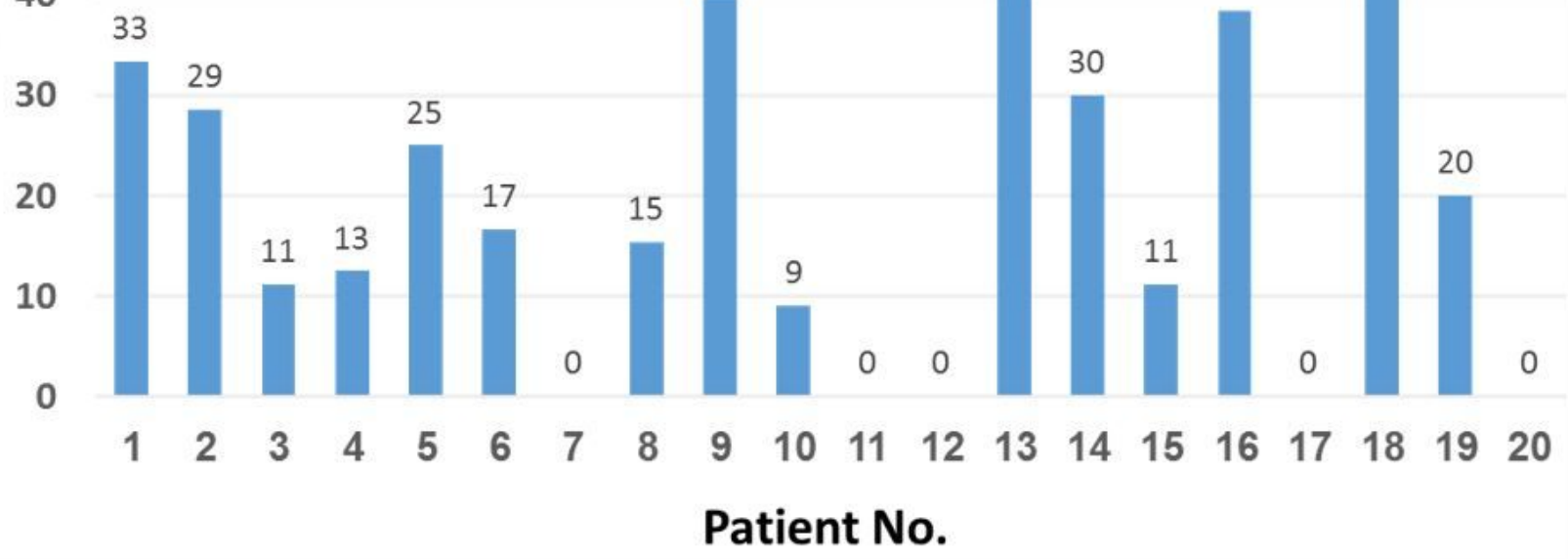

Figure 5

Reflux incidence after Fluid Bolus

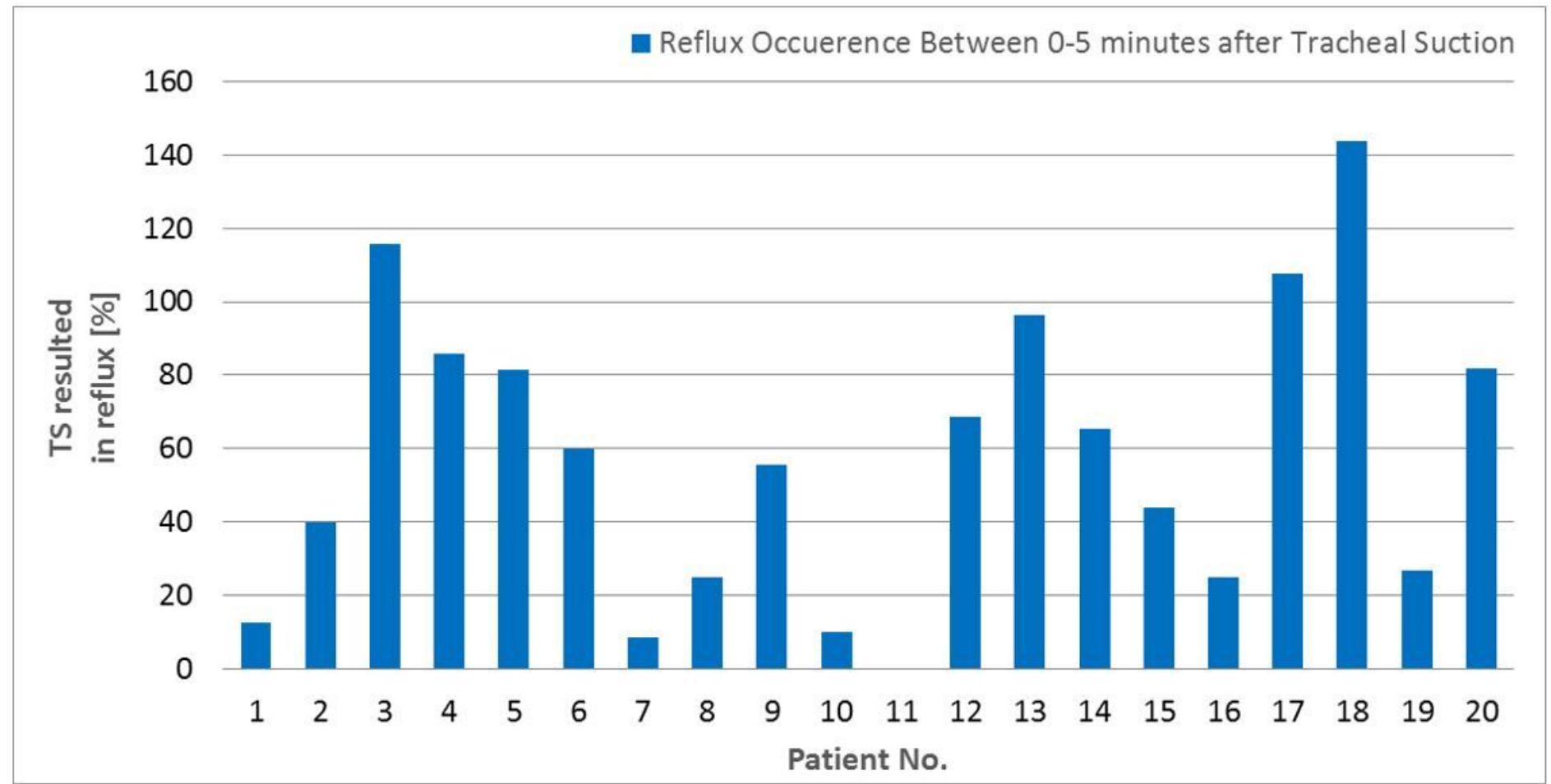

Figure 6

Tracheal suction to reflux correlation (reflux occurred within 0-5 min. of TS) 